\title{
A Unique of Rhizobium radiobacter Peritoneal Dialysis Catheter-related Peritonitis from a Cat Bite
}

\author{
Benjamin Bluen $^{1, *}$, Lukasz Kiljanek², Larry Krevolin², Kyle Krevolin ${ }^{3}$, Hans P. Schlecht ${ }^{1}$ \\ ${ }^{1}$ Drexel University College of Medicine, Division of Infectious Disease and HIV Medicine, \\ Hahnemann University Hospital, Philadelphia PA 19102 \\ Drexel University College of Medicine, Division of Nephrology, Hahnemann University Hospital, Philadelphia PA 19102 \\ ${ }^{3}$ Division of Clinical Microbiology, Hahnemann University Hospital, Philadelphia PA 19102 \\ *Corresponding author: bbluen@gmail.com
}

\begin{abstract}
Peritoneal dialysis catheter-related peritonitis is typically related to catheter insertion or hygiene. Organisms usually manifest as gram positive organisms typically Staphylococci or gram negative bacilli that typically colonize aqueous environments such as Pseudomonas spp. as well as other unusual mycobacterial or fungal pathogens. The authors present a unique case of peritoneal dialysis catheter-related peritonitis manifesting after the patient's cat bit the proximal aspect of the catheter attachment tubing. After failure to clinically improve after outpatient treatment of Staphylococcus hemolyticus and Staphylococcus capitis catheter related-peritonitis with intraperitoneal Vancomycin and empiric Gentamicin, repeat peritoneal cultures were sent that demonstrated growth of Rhizobium radiobacter. The patient was admitted inpatient and treatment was tailored to intraperitoneal Cefepime with clearance of peritoneal cultures and rapid improvement in peritoneal white blood cell count and the patient's clinical condition. The patient was subsequently discharged on oral Ciprofloxacin with complete recovery and catheter salvage.
\end{abstract}

Keywords: peritoneal dialysis, Rhizobium radiobacter, peritonitis, intraperitoneal, sensitivity

Cite This Article: Benjamin Bluen, LukaszKiljanek, Larry Krevolin, Kyle Krevolin, and Hans P. Schlecht,"A Case of Rhizobium radiobacter Peritoneal Dialysis Catheter-related Peritonitis from a Cat Bite." American Journal of Medical Case Reports, vol. 6, no. 6 (2018): 103-105. doi: 10.12691/ajmcr-6-6-1.

\section{Introduction}

Rhizobium radiobacter, a formerly reclassified from Agrobacterium class by 16s RNA analysis [1], is an aerobic gram-negative, motile, non-spore forming oxidase positive bacillus. Natural habitats for the bacteria exist in plants and soil [2]. Initially regarded as a contaminant, the organism was first determined in 1980 to cause prosthetic aortic valve endocarditis [3]. Previous case series have demonstrated the bacteria to be an opportunistic pathogen in patients with chronic indwelling catheters and hematologic malignancies. To this date, there are no reported cases of transmission of $R$. radiobacter via cat exposure. Due to the infrequent incidence of $R$. radiobacter, large clinical trials assessing its impact and treatment options are lacking.

\section{Case Presentation}

A 38 year-old female with end-stage renal disease on intermittent nocturnal peritoneal dialysis via Tenkhoff catheter for over two years secondary to type-1 diabetes mellitus presented to Hahnemann University Hospital in Philadelphia, Pennsylvania in October 2016 upon her nephrologist's request due to positive IP cultures. Her symptoms began several days after her cat bit her peritoneal dialysis (PD) connection tubing just distal to the Tenkhoff catheter attachment (see Figure 1). She subsequently developed nausea, vomiting, and abdominal discomfort that lasted 1-2 days. Outpatient dialysis cultures were initially positive for Staphylococcus hemolyticus and Staphylococcus capitis with white blood cell count demonstrating 90 cells/uL and $27 \%$ neutrophils. The patient was subsequently treated with three doses of IP Vancomycin and one initial dose of IP Gentamicin over the course of nine days. Two days later, due to persistent fatigue, decreased energy, and decreased appetite, repeat outpatient peritoneal cultures were taken that were positive for gram-negative bacilli. The patient was contacted and referred to the hospital for further evaluation.

After admission, the patient initially underwent treatment with IP vancomycin and gentamicin (Dianeal PD-2 Intraperitoneal dialysis solution with $2 \mathrm{~L}$ of $1.5 \%$ dextrose, $8 \mathrm{meQ} \mathrm{KCl}$ additive, $4 \mathrm{mg}$ gentamicin and 40mg vancomycin) for 3 days and intravenous cefepime dosed at $2 \mathrm{~g}$ q48 hours for 3 days. IP infusion dwell-time was set to 2.5 hours on a q3 hour basis. Initial inpatient peritoneal fluid analysis indicated WBC count of 274 cells/uL, 49\% neutrophils, confirming the diagnosis of peritoneal catheter-related peritonitis. Peritoneal culture repeated while inpatient on 5\% sheep's blood revealed gram-negative bacilli. Speciation by Vitek 2 (BioMérieux, Inc., Durham, NC) demonstrated Rhizobium radiobacter (see Figure 2, Figure 3). Subsequent peritoneal fluid analysis the next day revealed 
a WBC count of 9 cells/uL with $5 \%$ neutrophils with no demonstrated culture growth (see Table 1). Sensitivity analysis confirmed susceptibility to cefepime, ciprofloxacin, and piperacillin-tazobactam (see Table 2) and the patient's antibiotics were changed via infectious disease consult to IP cefepime $125 \mathrm{mg} / \mathrm{L}$ (Dianeal PD-2 Intraperitoneal dialysis solution with $2 \mathrm{~L}$ of $1.5 \%$ dextrose, $8 \mathrm{meQ} \mathrm{KCl}$ additive). The patient's symptoms gradually improved with this regimen and was discharged on oral ciprofloxacin $500 \mathrm{mg}$ daily for a 3 week treatment course with PD catheter retention.

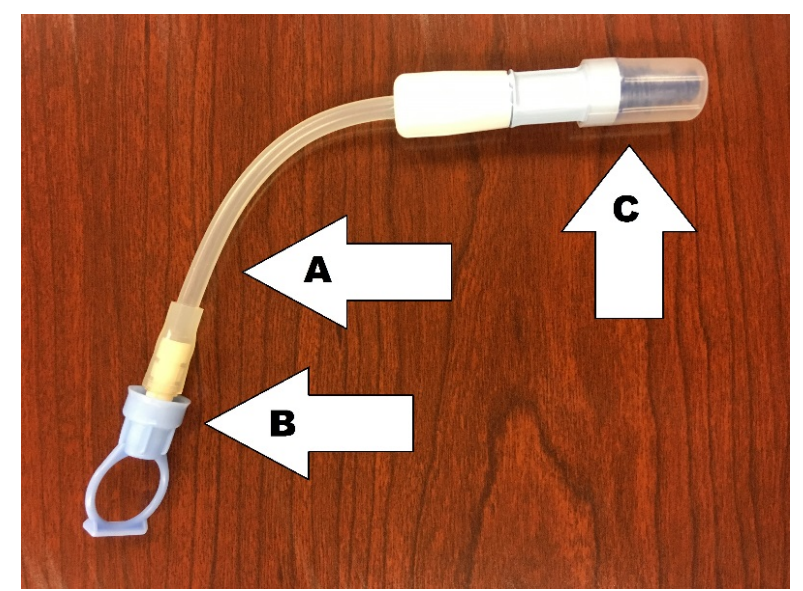

Figure 1. Peritoneal dialysis catheter demonstrating bite site (A). PD attachment site (B) and tubing connector (C) are noted

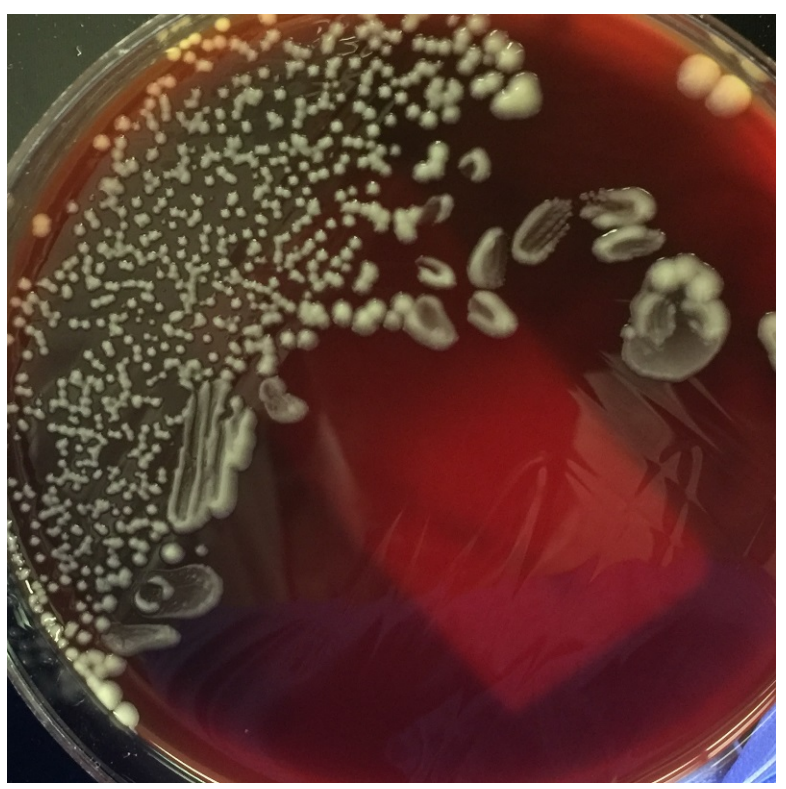

Figure 2. Colonies of $R$. radiobacter on blood agar

Table 1. Results of peritoneal fluid and culture

\begin{tabular}{|c|c|c|c|c|}
\hline $\begin{array}{c}\text { Peritoneal } \\
\text { Fluid }\end{array}$ & $\begin{array}{c}\text { 9 Days Prior to } \\
\text { Admit }\end{array}$ & $\begin{array}{c}2 \text { Days Prior } \\
\text { to Admit }\end{array}$ & $\begin{array}{c}\text { Day 1, } \\
\text { Inpatient }\end{array}$ & $\begin{array}{c}\text { Day 2, } \\
\text { Inpatient }\end{array}$ \\
\hline RBC & Not Reported & $\begin{array}{c}\text { Not } \\
\text { Reported }\end{array}$ & 1054 & 1 \\
\hline WBC & 90 & 181 & 274 & 9 \\
\hline Neutrophils & $27 \%$ & $56 \%$ & $49 \%$ & $5 \%$ \\
\hline & $\begin{array}{c}\text { Staphylococcus } \\
\text { epidermidis, } \\
\text { Staphylococcus } \\
\text { haemolyticus }\end{array}$ & $\begin{array}{c}\text { Rhizobium } \\
\text { radiobacter }\end{array}$ & $\begin{array}{c}\text { Rhizobium } \\
\text { radiobacter }\end{array}$ & $\begin{array}{c}\text { No } \\
\text { Growth }\end{array}$ \\
\hline
\end{tabular}

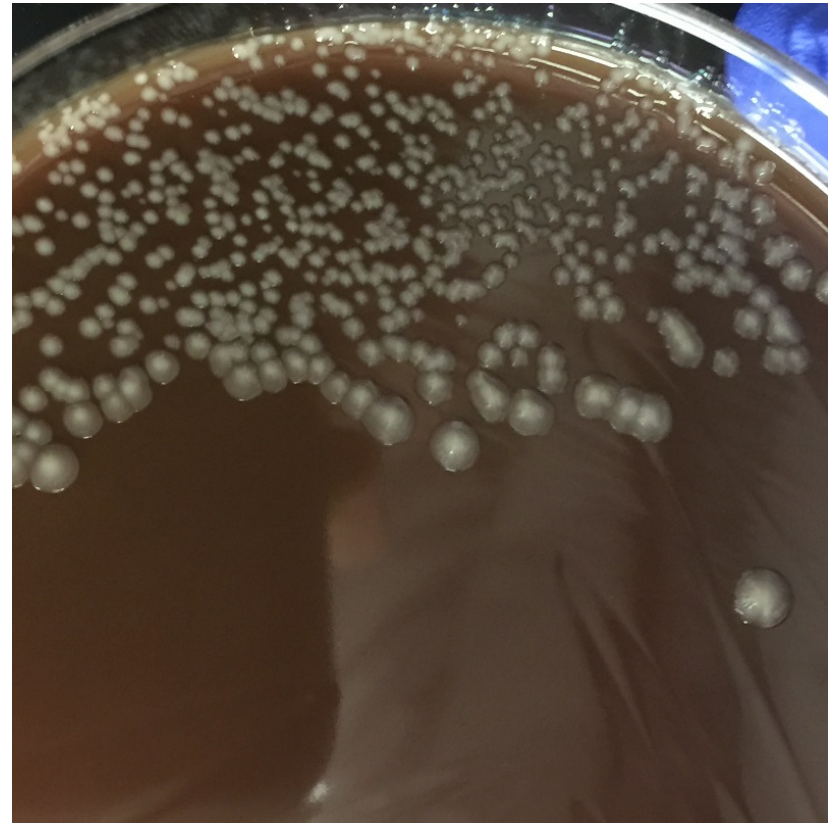

Figure 3. $R$. radiobacter growth on chocolate agar

Table 2. MIC sensitivities of $\boldsymbol{R}$. radiobacter isolate

\begin{tabular}{|l|c|c|}
\hline Antibiotic & MIC Value & Interpretation \\
\hline Aztreonam & 16 & Intermediate \\
\hline Cefepime & $<2$ & Sensitive \\
\hline Ciprofloxacin & $<0.5$ & Sensitive \\
\hline Gentamicin & 4 & Sensitive* \\
\hline Imipenem & $<1$ & Sensitive \\
\hline Levofloxacin & $<1$ & Sensitive \\
\hline Meropenem & $<1$ & Sensitive \\
\hline Piperacillin & $<16$ & Sensitive \\
\hline Ticarcillin/Clavulinate & $<8$ & Sensitive \\
\hline Tobramycin & 16 & Resistant \\
\hline Trimethoprim/Sulfamethoxazole & $>80$ & Resistant \\
\hline
\end{tabular}

*Gentamicin is commonly reported sensitive at this range, although the same effect is not observed in vivo.

\section{Discussion}

Rhizobium radiobacter is an infrequent cause of opportunistic infection largely of indwelling catheters and foreign material. The largest case-series involved 13 Taiwanese patients with $R$. radiobacter infection, of which the majority had chronic indwelling catheters. All isolates were susceptible to cefepime, ciprofloxacin, piperacillin-tazobactam, and carbapenems [4]. Although treatment frequently required foreign device removal [5], recent reports indicate that early and appropriate antibiotic therapy may allow catheter salvage in catheter-related $R$. radiobacter peritonitis [6]. $R$. radiobacter isolates should be evaluated in the appropriate clinical context as pseudo-outbreaks have been reported due to nonsterile processing equipment and/or handling of laboratory specimens [7]. As two separate laboratories confirmed $R$. radiobacter by culture and reactive peritoneal cell counts in our patient, true infection was confirmed.

This case documents the first reported transmission of $R$. radiobacter via cat exposure. The patient's cat may have been colonized with $R$. radiobacter while being near 
plants and soil at or nearby the patient's home. Infections transmitted by cat bites tend to be polymicrobial, including Staphylococci, Streptococci, Corynebacterium, Capnocytophagia, Pasteurella, and anaerobic flora [8]. A 1997-2013 literature review by Poliquin et al. found 30 cases of PD catheter-related peritonitis resulting from Pasteurella with cats being the most common source animal. Appropriate treatment and catheter retention was generally obtained with two weeks of amoxicillinclavulinate or IP cefazolin [9].

The revised 2016 International Society of Peritoneal Dialysis lists pets as a modifiable risk factor for PD catheter-related peritonitis [10]. As zoonotic injuries constitute approximately $1 \%$ of emergency department visits and \$30 million US dollars yearly, clinicians and patients alike should be aware of potential complications of pet-related interactions with indwelling medical devices [11].

\section{Conclusion}

Although uncommon, $R$. radiobacter should be considered in the differential diagnosis of potential pathogens in zoonotic peritoneal catheter-related peritonitis. The importance of patient pet owner education in taking measures to prevent zoonotic infections cannot be overstated.

\section{Acknowledgements}

The authors would like to thank the department of nephrology for the dedicated work of the peritoneal dialysis nursing staff. We would also like to acknowledge the contributions of the members of the microbiology laboratory whose efforts to identify pathogens is vital to daily patient care. The authors declare that they did not receive funding for this report.

\section{References}

[1] Young JM, Kuykendall LD, Martinez-Romero E, Kerr A, Sawada H. A revision of Rhizobium Frank 1889, with an emended description of the genus, and the inclusion of all species of Agrobacterium Conn 1942 and Allorhizobium undicola de Lajudie et al. 1998 as new combinations: Rhizobium radiobacter, R. rhizogenes, R. rubi, R. undicola and R. vitis. Int J Syst Eval Microbiol. 2001 Jan; 51(Pt 1): 89-103.

[2] Edmond MB, Riddler SA, Baxter CM, Wicklund BM, Pasculle AW. Agrobacterium radiobacter: A Recently Recognized Opportunistic Pathogen. Clin Infect Dis. (1993) 16 (3): 388-391.

[3] Plotkin GR. Agrobacterium radiobacter prosthetic valve endocarditis. Ann Intern Med 1980; 93: 839-40.

[4] Lai CC, Teng LJ, Hsueh PR, Yuan A, Tsai KC, Tang JL, Tien HF. Clinical and Microbiological Characteristics of Rhizobium radiobacter Infections. Clin Infect Dis. (2004) 38 (1): 149-153.

[5] Blumberg DA, Cherry JD. Agrobacterium radiobacter and CDC group Ve-2 bacteremia. Diagn Microbiol Infect Dis. 1989 Jul-Aug; 12(4): 351-5.

[6] Tsai SF. Rhizobium Radiobacter Peritonitis Revisited: Catheter removal Is Not Mandatory. Perit Dial Int May-June 2013vol. 33 no. 3 331-332.

[7] Pereira LA, Chan DSG, Ng MT, Lin R, Jureen R, Fisher DA, Tambyah P. Pseudo-Outbreak of Rhizobium radiobacter Infection Resulting from Laboratory Contamination of Saline Solution. J. Clin. Microbiol. July 2009 vol. 47. no. 7. 2256-2259.

[8] Ellis R, Ellis C. Dog and Cat Bites. Am Fam Physician. 2014 Aug 15; 90(4): 239-243.

[9] Poliquin PG, Lagace-Wiens P, Verrelli M, Allen DW, Embil JM. Pasteurella species peritoneal dialysis-associated peritonitis: Household pets as a risk factor. Can J Infect Dis Med Microbiol. 2015 Jan-Feb; 26(1): 52-55.

[10] Li PKTM et al. ISPD Peritonitis Recommendations: 2016 Update On Prevention And Treatment. Perit Dial Int 2016pdi.2016.00078.

[11] Griego RD, Rosen T, Orengo IF, Wolf JE. Dog, cat, and human bites: A review. J Am Acad Dermatol 1995 Dec; 33(6): 1019-29. 